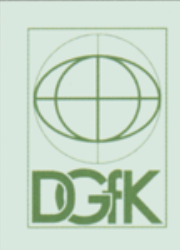

KN Kartographische Nachrichten Journal of Cartography and Geographic Information 68. Jahrgang, Juni 2018, Heft 3

Herausgeber: Deutsche Gesellschaft für Kartographie e.V. (DGfK) - Gesellschaft für Kartographie und Geomatik, vertreten durch den Präsidenten Prof. Dr. Manfred Weisensee

Organ der Deutschen Gesellschaft für Kartographic e.V. (DGFK). der Schweizerischen Gesellschaft für Kartografie (SGK) und der Österreichischen Kartographischen Kommission (OKK) in der Osterreichischen Gesellschaft für Geographie (ÖGG).

Schriftleitung

Hauptschriftleiter

Prof. Dr. rer. nat. habil. Mark Vetter (zuständig für Aufsătze, Berichte). Hochschule für angewandte Wissenschaften Würzburg-Schweinfurt, Münzstraße 12, 97070 Würzburg: vetter@kartographische-nachrichten.de

Fon: 0049(0) 931 3511-8249

Weitere Schriftleiter

Prof. Dr. phil. Frank Dickmann (Zweiter Schriftleiter, zuständig für Nachrichten aus Hochschulen und Institutionen, Rezensionen).

Nachrichten aus Hochschulen und institutionen, Rezen

Ruhr-Universităt Bochum, Geographisches Institut,
Postfach, 44780 Bochum; frank.dickmann@rub.de

Postfach, 44780 Bochum; frank.dickmann @rub.de

Dipl.-Ing. Andreas Gollenstede (zuständig für Geoinformation

aktuell) Haareneschstr. 91, 26121 Oldenburg; kn@gollenstede.com Fon: 0049(0) 441 7779-545; Fax: 0049(0) 441 7779-750

Dr--Ing. Thomas Chudy (zuständig für DGFK-, SKG- und ÖGG-

Nachrichten). Martin-Luther-Universităt Halle-Wittenberg, Institut

für Agrar- und Ernährungswissenschaften, Karl-Freiherr-von-

Fritsch-StraBe 4, 06120 Halle/Saale, Fon: 0049(0) 3455522448 :

Fax: 0049(0) 3455527109

Prof. Dr. Reinhard Zolitz (zuständig für Nachrichten aus Hochschulen und Institutionen), Institut für Geographie und Geologie, Kartographie und GIS, Friedrich-Ludwig-Jahn-Str. 16,

D-17489 Greifswald, Fon 03834864523, Fax: 03834864501,

E-Mail: zoelitz@uni-greifswald.de

Mitarbeit für die Schweiz: Prof. Dr. Lorenz Hurni. Institut für Kartografie, ETH Hōnggerberg. 8093 Zürich; hurni@karto.baug.ethz.

ch. Fon: 004116333033 ; Fax: 00411631153

Mitarbeit für Osterreich: Univ-Prof. Dr. Wolfgang Kainz Institut für Geographie und Regionalforschung. Universitāt Wien, Universitätsstrafe 7/5, 1010 Wien: wolfang kainzeunivie

Fon: 0043 14277-8640; Fax: 0043 14277-9531

Ehrenschriftleiter

Prof. Dr. Jürgen Dodt t, Witten

Dr. rer. nat. h. c. Rolf Harbeck, Bonn

Editorial Board

Prof. Dr. H. Asche, Potsdam; Prof. Dr. Manfred Buchroithnec,

Dresden; Prof. Dr. Dirk Burghardt, Dresden; Prof. Dr. D. Dransch,

Potsdam; Prof. Dr. M. Ehlers, Osnabrück, Berlin; Prof. Dr. S. I.

Fabrikant, Zürich, Schweiz; Ass-Prof. Dr. S. Fuhrmann, Fairfax

USA; Prof. Dr. G. Gartner, Wien, Osterreich; Prof. Dr. D. Grünreich Ronnenberg; Dr. Rolf Harbeck, Bonn; Prof. Dr. A. Hüttermann, Ludwigsburg; Prof. Dr. L. Hurni, Zürich; Prof. Dr. W. Kainz, Wien: Prof. Dr. P. Kammerer, München; Prof. Dr. W. G. Koch, Dresden; Prof. Dr. Menno-Jan Kraak, Twente, Netherlands: Prof. Dr. Jukka Krisp, Augsburg; Prof. Dr.-Ing. L. Meng. München:

Prof. Dr. J. Schiewe, Hamburg: PD Dr.-Ing. J. Schoppmever, Bonn: Prof. Dr. J. Schiewe, Hamburg; Po Dr.--Ing. S. Schooppmeyer, Bonn: Prof. Dr. J. Siemer, Regina, Kanada; Prof. Dr. R. Zölitz, Greifswald. Manuskripte richten Sie bitte an den zuständigen Schriftleiter. Hinweise zur Gestaltung des Manuskripts finden Sie unter www.kartographische-nachrichten.de > Autorenhinweise. Reviewverfahren

Wissenschaftliche Beiträge werden im Rahmen eines Peer-ReviewVerfahrens begutachtet. Trotz sorgfältiger Redigierung übernehmen Herausgeber, Redaktion und Verlag keine Haftung für die inhaltliche Herausgeber, Redaktion und Verlag
Richtigkeit der Veröffentlichungen.

Die KN Kartographische Nachrichten ist in der internationalen Zitationsdatenbank Scopus gelistet

Die DGfK ist Mitglied der

Internationalen Kartographischen

Vereinigung IKV/ICA

Verlag: Kirschbaum Verlag $\mathrm{GmbH}$

Siegfriedstraße 28, D-53179 Bon

Postfach 210209, D-53157 Bonn

Fon: (0228) 95453-0, Fax: (0228) 95453-27

Internet: $h$ ttp://www.kirschbaum.de, E-Mail: info@kirschbaum.de

Anzeigenleiter: Michael Dietl Nerlagsleiter Fachzeitschriften prin

\&t digital), Fon: (0228) 95453-47, E-Mail: m.diet|@kirschbaum.de;

Anzeigenpreisliste Nr. 53 vom 1.10.2017

Bezugspreise und ISSN: Inland/Ausland Jahresabonnement

inkl. E-Paper und elektronischem Archiv 74 - Euro zzgl.

Versand 9,80,- Euro (jeweils einschl. MwSt.).

Einzelheft 18,- Euro zzgl. Versand

Die Zeitschrift erscheint alle zwei Monate. ISSN 0022-9164.

Kündigungsfrist 6 Wochen zum Auslaufen des Abonnement-

Zeitraumes

Satz: EMS Eckert Medienservice, 53881 Euskirchen

Druck: johnen-druck GmbH \& CO. KG. Industriegebiet Bornwiese.

54470 Bernkastel-Kues

\section{Neogeographie - wenn Nicht-Kartographen Karten machen}

\section{Liebe Leserinnen und Leser,}

am 16. und 17. November 2017 fand in Leipzig die Abschlusstagung eines Forschungsprojekts statt, das der Frage nachging, wie kartographisch nicht ausgebildete Personen Karten in neuen Medien produzieren und in welchem Maß sie kritisch mit solchen Darstellungen umgehen. Im Verlauf der vergangenen vier Jahre

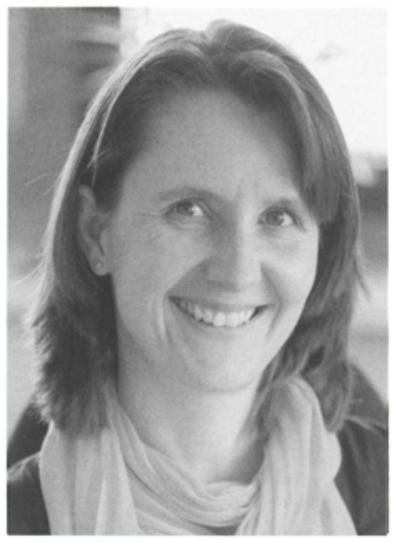

Jana Moser forschte das interdisziplinär zusammengesetzte Projektteam unter Nutzung des Begriffs der Prosumer (als Bezeichnung der Gleichzeitigkeit von Produzent und Konsument) zu Fragen von Produktionsbedingungen und -kontexten, technologischen Abhängigkeiten, Darstellungsmethoden, der Veränderung von Weltbildern und der kritischen Auseinandersetzung mit Webkarten. Darüber hinaus standen und stehen weiterhin aber auch konzeptionelle und forschungsmethodische Fragen von Webkartographie im Fokus des Interesses. Diese und weitere Fragen wurden im Rahmen der erwähnten Tagung unter der bewusst provokanten Frage „Neogeographie - Kompetente Partizipation oder Illusion von Demokratisierung?“ mit etwa 50 Teilnehmern intensiv diskutiert.

In diesem Themenheft wird eine Auswahl der Ergebnisse der Tagung vorgestellt. Das Konzept „Neogeographie“ umreißt dabei vor allem die Bandbreite des Themas und kartographischen Forschungsfeldes. Einführend erläutert der Beitrag von Jana Moser Fragestellungen und Hintergründe von „Neogeographie“ und „Neokartographie“ und thematisiert Chancen und Herausforderungen für die kartographische Forschung. Christian Bittner und Georg Glasze untersuchen am Beispiel kollaborativer Kartierungsplattformen verschiedene Exklusionsmechanismen und deren Einfluss auf die Generierung von Wissen. Übrigens entstand das Umschlagbild dieser KN-Ausgabe als Livezeichnung während des Vortrages der beiden Autoren und visualisiert verschiedene (auch räumliche) Dimensionen von Exklusion und Inklusion auf eine Weise, die für Kartographen zunächst ungewohnt ist. Exklusions- und Inklusionsmechanismen in Bezug auf Bildungsansätze und didaktische Konzepte zum Umgang mit Geomedien werden auch im Beitrag von Jana Pokraka und Inga Gryl kritisch analysiert. Demgegenüber untersucht der Beitrag von Sebastian Meier und Katrin Glinka Beispiele von Diensten und Forschungsprojekten, die die Analyse, aber auch kritische Auseinandersetzung mit selbst generierten raumbezogenen Daten wie täglichen Bewegungsprofilen erlauben. Auch beim Bergwandern entstehen digital erfassbare Bewegungsprofile in Form von Routen. Welche Medien Bergsportler für ihre Aktivitäten bevorzugt nutzen und in welchen Stadien analoge oder digitale Produkte präferiert werden, sind Themen des Beitrages von Julia Moritz, Magdalena Novak und Stephan Schwan.

Ich würde mir wünschen, dass dieses Themenheft die weiterhin bestehenden Potenziale von Neogeographie für die Kartographie verdeutlicht und eine weitere - auch kritische - Auseinandersetzung mit den aktuellen technischen, konzeptionellen, methodischen aber auch bildungspolitischen Aspekten von Webkartographie anregt.

Herzlichst, Ihre

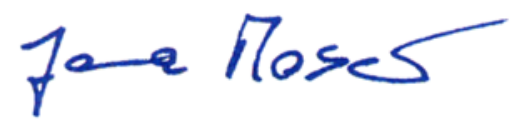

